\title{
An Aberration Corrected FIB for Nano-Area Mass Spectrometry
}

S. Itose, ${ }^{*}$ M. Matsuya, ${ }^{*}$ S. Uno, $*$ K. Yamashita, ${ }^{*}$ S. Ebata, **, M. Ishihara, **, K. Uchino, *** H. Yurimoto, $* * * *$ K. Sakaguchi, * and M. Kudo*

* JEOL Ltd, 1-2 Musashino, 3-Chome, Akishima, Tokyo, 196-8558, Japan.

** Osaka University, 1-1 Machikaneyama, Toyonaka, Osaka, 560-0043, Japan.

*** Kyushu University, 6-1 Kasuga-koen, Kasuga-city, Fukuoka, 816-8500, Japan

**** Hokkaido University, Kita-ku, Sapporo, Hokkaido, 060-0810, Japan

Introduction: A focused ion beam system (FIB) with an aberration corrector has been developed for Laser Ionization Mass Nanoscope (LIMAS) [1]. Analysis of meteorites or substances brought back by a spacecraft is very important to survey the formation of the solar system and the universe. This requires the measurement of isotopes in fine structures of the specimens [2] under the condition of so limited amount of the specimens. We will present an aberration corrected FIB for this purpose.

Instrumentation: Fig. 1 shows an overview of the LIMAS. An FIB with a $\mathrm{Ga}^{+}$liquid-metal ion source (LIMS) is equipped with the aberration corrector which is composed of the electrostatic 8 dodecapoles [3]. Optical conditions are optimized for both cases in which the corrector is turned on and off as shown in Fig. 2. The corrector can correct both geometric and chromatic aberrations, and the diameter of the ion probe is decreased to about $1 / 2-1 / 3$ by the corrector for the probe current (Ip) range $1 \mathrm{pA}-10 \mathrm{nA}$. The sputtered neutrals from the small area with the same size of the probe diameter are ionized near the specimen surface by the $800 \mathrm{~nm}$ photons with a peak intensity of 75 GW from a laser system with the pulse width of 40 femto seconds. To reduce the background ions, the pressure of the specimen chamber is kept less than $1 \times 10^{-7} \mathrm{~Pa}$. These so-called post ions and the secondary ions are extracted and injected to the multi turn mass spectrometer named MULTUM [4] with high resolving power better than $10^{4}$.

Results: Operating conditions for the corrector were set for each accelerating voltage and probe current from the stored data files. A semi-automatic aberration correction procedure assisted the fine-tuning of the optics. Fig. 3 shows an example of the scanning ion microscope (SIM) images of gold particles on a graphite block for both cases using ion beam energy $20 \mathrm{keV}$ and Ip $=100 \mathrm{pA}$. The image with the corrector was obviously clearer than that without the corrector. The effect of the correction and residual aberrations were verified from the beam shapes by taking the images for the under and the over focus [5]. We expect that the LIMAS will serve the analysis of the specimens of Asteroid Itokawa which were brought back by the asteroid explorer "HAYABUSA."

\section{References}

[1] S. Ebata, et al, submitted to ASMS2011, to be published.

[2] J. P. Greenwood, et al, Nature Geoscience 4 (2011) 79.

[3] C. Weißbäcker and H. Rose, J. Electron Microsc. 50 (2001) 383; 51 (2002) 45.

[4] D. Okumura, et al, Nucl. Instr. Meth. in Phys. Res. A 519 (2004) 331.

[5] S. Uno, K. Honda, N. Nakamura, M. Matsuya and J. Zach, Optik 116(2005) 438.

[6] This work was partially supported by a grant from the "System Development Program for

Advanced Measurement and Analysis" of the Japan Science and Technology Agency (JST).

We thank Dr. Zach in CEOS for the joint development of a proto-type corrector system. 


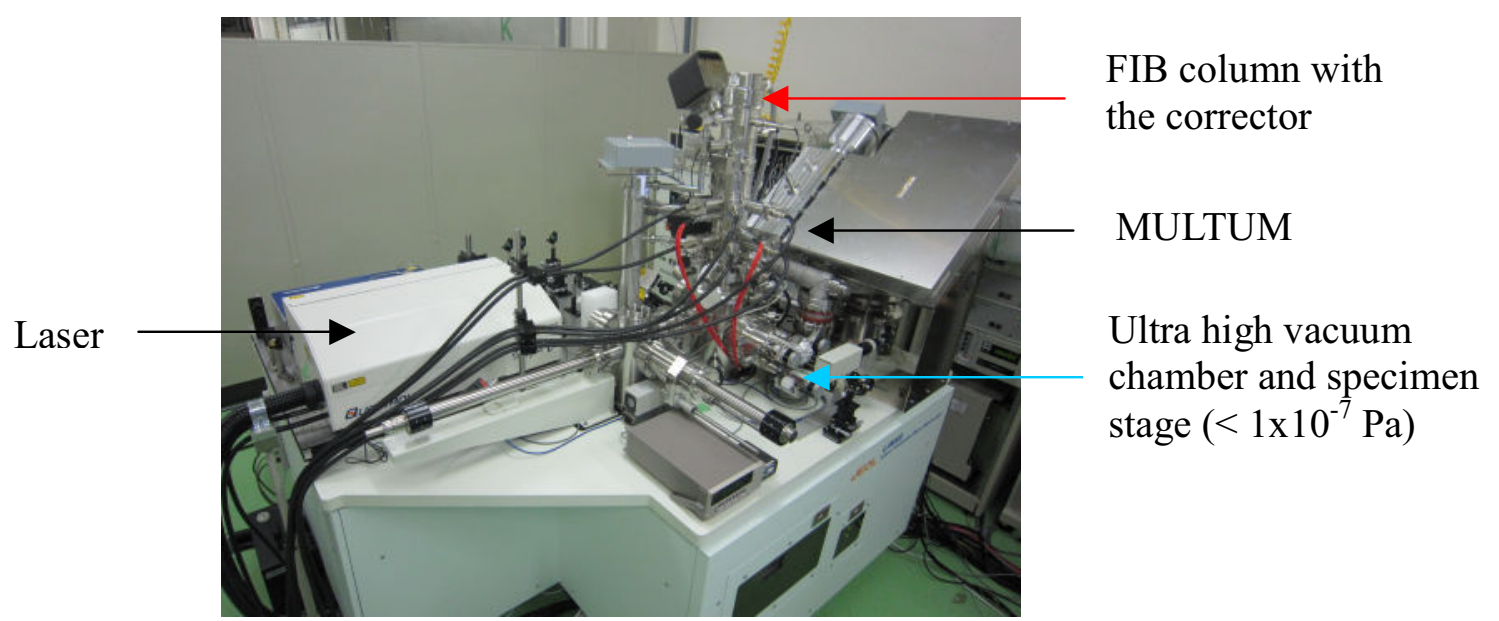

Fig. 1. Overview of the LIMAS equipped with an aberration corrector.
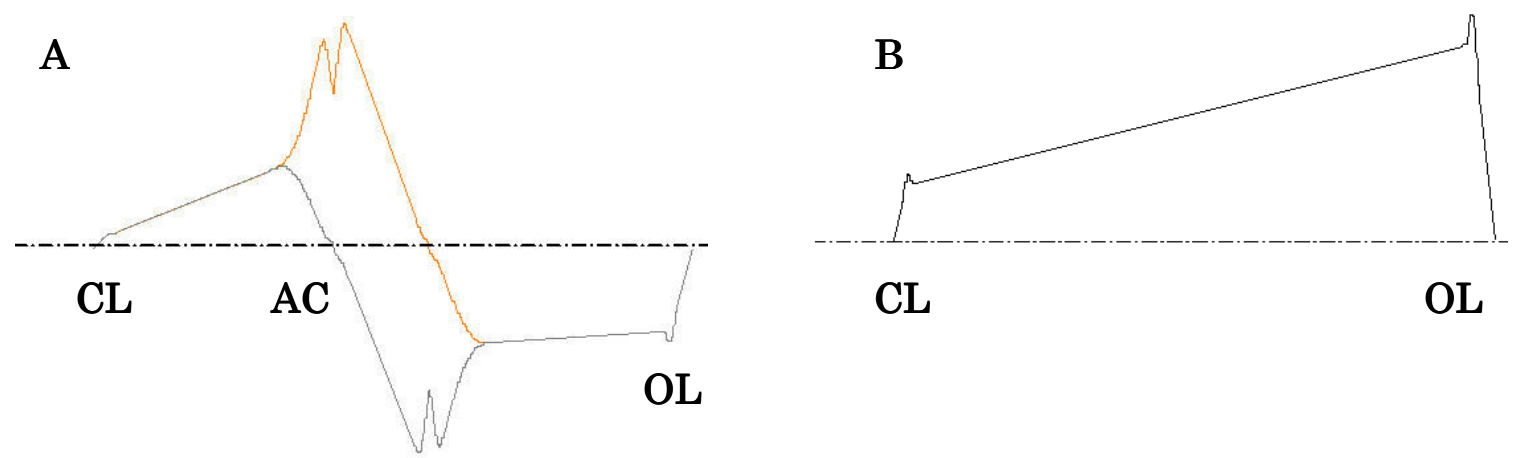

Fig. 2 Schematic ray path for a corrected FIB system. CL: Condenser lens. AC: Aberration corrector. OL: Objective lens. A. With the corrector.

B. Without the corrector.
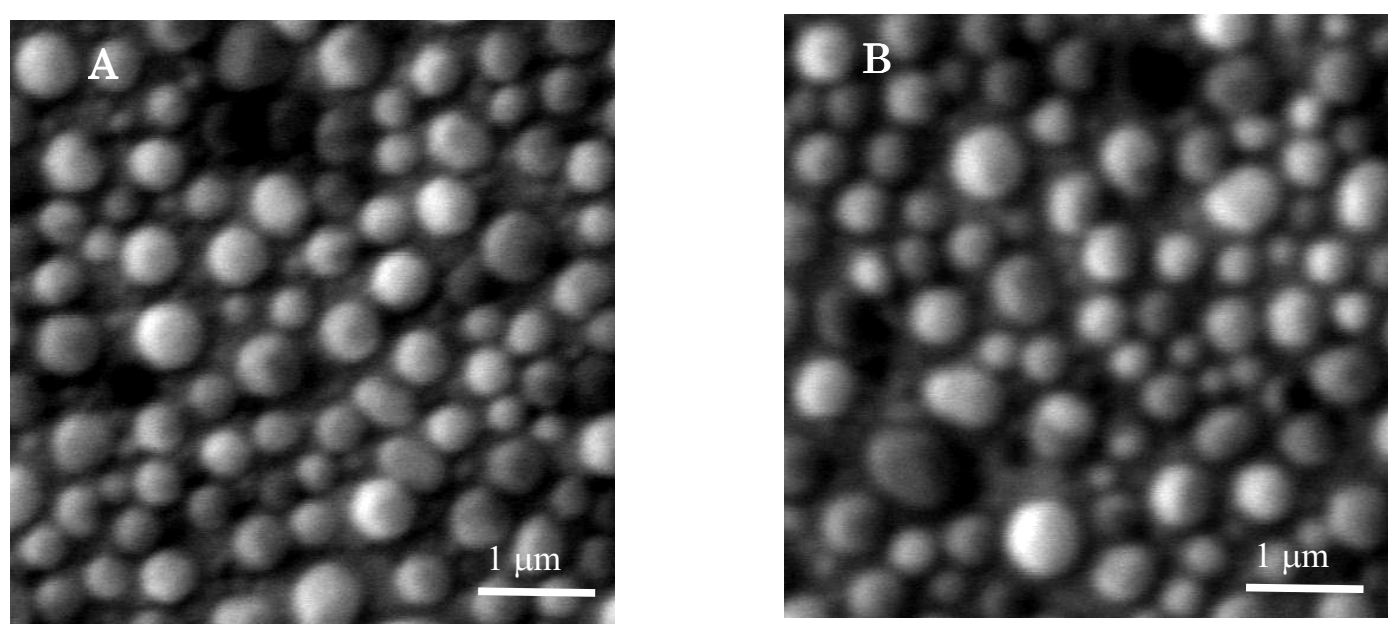

Fig. 3. SIM images of Au particles by the secondary electrons. $20 \mathrm{keV}$, Ip $=100 \mathrm{pA}$.
A. With the corrector.
B. Without the corrector. 